PROCEEDINGS OF THE

AMERICAN MATHEMATICAL SOCIETY

Volume 140, Number 9, September 2012, Pages 3145-3150

S 0002-9939(2012)11193-1

Article electronically published on January 10, 2012

\title{
A NOTE ON POINCARÉ'S PROBLEM FOR QUASI-HOMOGENEOUS FOLIATIONS
}

\author{
MAURÍCIO CORRÊA JR. AND MÁRCIO G. SOARES \\ (Communicated by Ted Chinburg)
}

\begin{abstract}
We consider the question of bounding the degree of curves which are invariant by a holomorphic foliation of a given degree on a well-formed weighted projective plane.
\end{abstract}

\section{Introduction AND STATEMENT OF RESUlt}

In this paper we consider the question of bounding the degree of curves which are invariant by a holomorphic foliation of a given degree on a well-formed weighted projective plane. This question had been considered by several authors ever since H. Poincaré raised it in a more specific setting, that of algebraic integration of polynomial differential equations in $\mathbb{C}^{2}$; see [1, [2], [5], [6], 10], [1] and [13].

We recall that, in the usual complex projective situation, the presence of dicritical singularities makes the question meaningless, as is shown by simple examples, such as Example 3 below and, in a much more involved and elucidating context, by the examples given by A. Lins Neto in [8].

However, denoting by $d^{o}$ and $d$ the degrees of the curve and of the foliation, respectively, in case the invariant curve is nonsingular we get the bound $d^{o} \leq$ $d+1$, which holds also in higher dimensions 13 regardless of the nature of the singularities of the foliation along the invariant variety. Also, as shown in [1, for curves with only nodal singularities, we get the same estimate in case it is irreducible, and $d^{o} \leq d+2$ in the reducible case.

We consider this question in $\mathbb{P}_{w}:=\mathbb{P}\left(w_{0}, w_{1}, w_{2}\right), w_{i} \in \mathbb{N}$ two-by-two coprimes, endowed with a singular holomorphic foliation which admits an invariant quasismooth curve. Let $\operatorname{Sing}\left(\mathbb{P}_{w}\right)$ and $\operatorname{Sing}(\mathcal{F})$ denote the singular sets of $\mathbb{P}_{w}$ and of $\mathcal{F}$, respectively. The result is as follows:

Theorem. Let $\mathcal{F}$ be a singular holomorphic foliation on $\mathbb{P}_{w}$, of degree $\operatorname{deg}(\mathcal{F})$, with $\operatorname{Sing}(\mathcal{F}) \cap \operatorname{Sing}\left(\mathbb{P}_{w}\right)=\emptyset$ and $C$ a quasi-smooth curve of degree $\operatorname{deg}(C)$ which is invariant by $\mathcal{F}$. Then,

$$
\operatorname{deg}(C) \leq \operatorname{deg}(\mathcal{F})+\frac{w_{0}+w_{1}+w_{2}-2}{w_{0} w_{1} w_{2}}
$$

Received by the editors September 24, 2009 and, in revised form, November 24, 2010 and March 22, 2011.

2010 Mathematics Subject Classification. Primary 32S65; Secondary 14M25.

Key words and phrases. Holomorphic foliations, weighted projective spaces.

The authors' work was partially supported by CNPq (Brasil).

(C)2012 American Mathematical Society Reverts to public domain 28 years from publication 
This bound cannot be improved, as is shown by

Example 1. Let $f(x, y, z)=x^{m k}+y^{m k}-z^{k}$ and $g(x, y, z)=a x^{m}+b y^{m}+c z$, $m, k \in \mathbb{N}$. These are quasi-homogeneous polynomials of type $(1,1, m)$ and degrees $\mathrm{km}$ and $m$, both defining quasi-smooth curves of degrees $k$ and 1, respectively, which avoid the singularity of $\mathbb{P}(1,1, m)$. The 1 -form $\eta=k f d g-g d f$ defines a singular foliation $\mathcal{F}$ on $\mathbb{P}(1,1, m)$ of degree $\operatorname{deg}(\mathcal{F})=k-1 / m$. The orbifold $C=(f=0)$ is $\mathcal{F}$-invariant and

$$
\operatorname{deg}(C)=k \leq k-\frac{1}{m}+1=\operatorname{deg}(\mathcal{F})+1 .
$$

Example 2. Let $f(x, y, z)=x^{m+1}+y^{m+1}-(x+y) z$ and $g(x, y, z)=a x^{m}+b y^{m}+c z$, $2 \leq m \in \mathbb{N}$. These are quasi-homogeneous polynomials of type $(1,1, m)$ and degrees $m+1$ and $m$, both defining quasi-smooth curves of degrees $1+1 / m$ and 1 . Note that the curve $C=(f=0)$ passes through the singular point $[0: 0: 1]_{(1,1, m)}$ of $\mathbb{P}(1,1, m)$ and is smooth there. The 1 -form $\eta=(1+1 / m) f d g-g d f$ defines a singular foliation $\mathcal{F}$ on $\mathbb{P}(1,1, m)$, of degree $\operatorname{deg}(\mathcal{F})=1$, which is nonsingular at $[0: 0: 1]_{(1,1, m)} . C$ is $\mathcal{F}$-invariant and

$$
\operatorname{deg}(C)=1+1 / m \leq 1+1=\operatorname{deg}(\mathcal{F})+1 .
$$

Example 3. Let $k$ be a positive integer and

$$
\eta=\frac{y z}{k} d x+\left(m-\frac{1}{k}\right) x z d y-x y d z .
$$

$\eta$ induces a singular foliation $\mathcal{F}$ on $\mathbb{P}(1,1, m)$ of degree $\operatorname{deg}(\mathcal{F})=1 / m$. The curve $C=\left\{z^{k}-x y^{k m-1}=0\right\}$ has degree $k$ and is $\mathcal{F}$ - invariant. Since $k$ is arbitrary, the theorem does not hold. Here we have $[0: 0: 1]_{(1,1, m)}$ as a singular point of the foliation and also, $[1: 0: 0]_{(1,1, m)}$ is a singularity of $C$ and a dicritical singularity of $\mathcal{F}$.

\section{Foliations on Weighted projective planes}

We start by recalling weighted projective planes. Let $w:=w_{0}, w_{1}, w_{2}$ be positive integers which are two-by-two coprimes. $\mathbb{C}^{*}$ acts on $\mathbb{C}^{3} \backslash\{0\}$ by $\lambda .\left(z_{0}, z_{1}, z_{2}\right)=$ $\left(\lambda^{w_{0}} z_{0}, \lambda^{w_{1}} z_{1}, \lambda^{w_{2}} z_{2}\right)$. The quotient space by this action is the weighted projective plane of type $w, \mathbb{P}\left(w_{0}, w_{1}, w_{2}\right):=\mathbb{P}_{w}$. In case $w_{i}>1$ for some $i, \mathbb{P}(w)$ is a compact algebraic surface with cyclic quotient singularities. Following [9], we have a natural orbifold map

$$
\begin{aligned}
f_{w}: \mathbb{P}^{2} & \longrightarrow \mathbb{P}_{w} \\
{\left[z_{0}: z_{1}: z_{2}\right] } & \longmapsto\left[z_{0}^{w_{0}}: z_{1}^{w_{1}}: z_{2}^{w_{2}}\right]_{w},
\end{aligned}
$$

which allows us to show that there exists a unique (up to isomorphism) rank 1 complex $\mathbb{Q}$-bundle $\mathcal{O}_{\mathbb{P}_{w}}(1)$ over $\mathbb{P}_{w}$, such that

$$
f_{w}^{*} \quad \mathcal{O}_{\mathbb{P}_{w}}(1) \cong \mathcal{O}_{\mathbb{P}^{2}}(1) .
$$

The Euler sequence carries over to the weighted case, and we have the exact sequence of $\mathbb{Q}$-bundles over $\mathbb{P}_{w}$ :

$$
0 \longrightarrow \mathbb{C} \longrightarrow \mathcal{O}_{\mathbb{P}_{w}}\left(w_{0}\right) \oplus \mathcal{O}_{\mathbb{P}_{w}}\left(w_{1}\right) \oplus \mathcal{O}_{\mathbb{P}_{w}}\left(w_{2}\right) \longrightarrow T \mathbb{P}_{w} \longrightarrow 0,
$$


where $\mathbb{C}$ is the trivial orbifold bundle and $T \mathbb{P}_{w}$ is the orbifold tangent bundle of $\mathbb{P}_{w}$. Also, see [9], the Chern-Weil theory of Chern classes holds as well in $\mathbb{P}_{w}$ as in projective spaces and, denoting by $\zeta=c_{1}\left(\mathcal{O}_{\mathbb{P}_{w}}(1)\right)$ we have, from (77),

$$
c\left(T \mathbb{P}_{w}\right)=\left(1+w_{0} \zeta\right)\left(1+w_{1} \zeta\right)\left(1+w_{2} \zeta\right)
$$

and hence

$$
c_{i}\left(T \mathbb{P}_{w}\right)=\sigma_{i}\left(w_{0}, w_{1}, w_{2}\right) \zeta^{i}
$$

where $\sigma_{i}$ is the $i$-th elementary symmetric function.

Now, let $X$ be a quasi-homogeneous vector field of type $\left(w_{0}, w_{1}, w_{2}\right)$ and degree $d$ in $\mathbb{C}^{3}$; that is, writing $X=\sum_{i=0}^{2} P_{i}(z) \frac{\partial}{\partial z_{i}}$ we have that $P_{i}\left(\lambda^{w_{0}} z_{0}, \lambda^{w_{1}} z_{1}, \lambda^{w_{2}} z_{2}\right)=$ $\lambda^{d+w_{i}-1} P_{i}\left(z_{0}, z_{1}, z_{2}\right)$. These descend well to $\mathbb{P}_{w}$. In fact, tensorize $(1)$ by $\mathcal{O}_{\mathbb{P}_{w}}(d-1)$ to get

$$
0 \longrightarrow \mathcal{O}_{\mathbb{P}_{w}}(d-1) \longrightarrow \bigoplus_{i=0}^{2} \mathcal{O}_{\mathbb{P}_{w}}\left(d+w_{i}-1\right) \longrightarrow T \mathbb{P}_{w} \otimes \mathcal{O}_{\mathbb{P}_{w}}(d-1) \longrightarrow 0
$$

It follows that a quasi-homogeneous vector field $X$ induces a foliation $\mathcal{F}$ of $\mathbb{P}_{w}$ and that $g R_{w}+X$ define the same foliation as $X$, where $R_{w}$ is the adapted radial vector field $R_{w}=w_{0} z_{0} \frac{\partial}{\partial z_{0}}+w_{0} z_{1} \frac{\partial}{\partial z_{1}}+w_{2} z_{2} \frac{\partial}{\partial z_{2}}$, with $g$ a quasi-homogeneous polynomial of type $\left(w_{0}, w_{1}, w_{2}\right)$ and degree $d-1$.

Dually, noting that $|w|=w_{0}+w_{1}+w_{2}$, we have the exact sequence

$$
0 \rightarrow \Omega_{\mathbb{P}_{w}}^{1} \otimes \mathcal{O}_{\mathbb{P}_{w}}(d+|w|-1) \rightarrow \bigoplus_{i=0}^{2} \mathcal{O}_{\mathbb{P}_{w}}\left(d+|w|-w_{i}-1\right) \rightarrow \mathcal{O}_{\mathbb{P}_{w}}(d+|w|-1) \rightarrow 0
$$

Hence, a foliation $\mathcal{F}$ of $\mathbb{P}_{w}$ is also induced by a 1 -form $\eta=A_{0} d z_{0}+A_{1} d z_{1}+A_{2} d z_{2}$, with $A_{i}$ a quasi-homogeneous polynomial of type $\left(w_{0}, w_{1}, w_{2}\right)$, degree $d+|w|-w_{i}-1$ and $\imath_{R_{w}} \eta=w_{0} z_{0} A_{0}+w_{1} z_{1} A_{1}+w_{2} z_{2} A_{2} \equiv 0$.

We shall assume that

$$
\operatorname{Sing}(\mathcal{F}) \cap \operatorname{Sing}\left(\mathbb{P}_{w}\right)=\emptyset
$$

The reason for this assumption is the following argument, given in 4]: around an orbifold point $p_{i} \in \operatorname{Sing}\left(\mathbb{P}_{w}\right)$ the surface is of the type $B^{2} / \Gamma_{i}$, where $B^{2}$ is the unit ball in $\mathbb{C}^{2}$ and $\Gamma_{i}$ is a $w_{i}$-cyclic group. Suppose we have a foliation $\mathcal{F}$ on $\mathbb{P}_{w} \backslash \operatorname{Sing}\left(\mathbb{P}_{w}\right)$ and consider the neighbourhood $U=B^{2} / \Gamma_{i}$ of $p_{i}$. Then we can lift $\mathcal{F}_{\mid U \backslash p_{i}}$ to $B^{2} \backslash\{0\}$, and on this covering the foliation can be defined by a holomorphic vector field which admits a holomorphic extension to $0 . \mathcal{F}$ is nonsingular at $p_{i}$ if this extension is nonvanishing at 0 . In this case the foliation is, after a $\Gamma_{i}$-equivariant holomorphic change of coordinates, the quotient of, say, the vertical foliation on $B^{2}$. Hence, the leaves are defined as in the smooth situation, by gluing the local leaves of $\mathcal{F}$ at regular points of the foliation and are therefore orbifolds.

We proceed now to define the "degree", $\operatorname{deg} \mathcal{F}$, of such a foliation. Recall that, in the usual projective situation, $\operatorname{deg} \mathcal{F}$ is the degree of the variety of tangencies of $\mathcal{F}$ with a generic hyperplane. 
An analogous geometric interpretation holds in the weighted situation, and we similarly have the corresponding canonical $\mathbb{Q}$-bundles $K_{\mathbb{P}_{w}}, K_{\mathcal{F}}$ and the $\mathbb{Q}$-bundles $T_{\mathcal{F}}, N_{\mathcal{F}}, N_{\mathcal{F}}^{*}$, all lying in $\operatorname{Pic}\left(\mathbb{P}_{w}\right) \otimes \mathbb{Q}$. The adjunction formula

$$
K_{\mathbb{P}_{w}}=K_{\mathcal{F}} \otimes N_{\mathcal{F}}^{*}
$$

still holds, and we point out that $K_{\mathbb{P}_{w}}=\mathcal{O}_{\mathbb{P}_{w}}(-|w|), K_{\mathcal{F}}=\mathcal{O}_{\mathbb{P}_{w}}(d-1)$ and $N_{\mathcal{F}}=$ $\mathcal{O}_{\mathbb{P}_{w}}(d+|w|-1)$.

Let $C$ be a compact connected curve (possibly singular) whose irreducible components are not $\mathcal{F}$-invariant. Then, for $p \in C$, the index $\operatorname{tang}(\mathcal{F}, C, p)$ is defined as in [3] and, writing $\operatorname{tang}(\mathcal{F}, C)=\sum_{p \in C} \operatorname{tang}(\mathcal{F}, C, p)$, we have that

$$
\operatorname{tang}(\mathcal{F}, C)=K_{\mathcal{F}} \cdot C+C \cdot C \geq 0 .
$$

We define the degree of $\mathcal{F}$ just as in the usual projective situation, that is,

$$
\operatorname{deg}(\mathcal{F}):=\operatorname{tang}(\mathcal{F}, H),
$$

where $H$ is a curve defined by a generic irreducible quasi-homogeneous polynomial of degree $w_{0} w_{1} w_{2}$.

After the work of I. Satake (see [9] and [12]), Poincaré's duality holds, so that $H$ can be seen as the Poincaré dual of $c_{1}\left(\mathcal{O}_{\mathbb{P}_{w}}(1)\right)$.

Before calculating (15) we recall that

$$
\int_{\mathbb{P}_{w}}^{o r b} c_{1}^{2}\left(\mathcal{O}_{\mathbb{P}_{w}}(1)\right)=\frac{1}{w_{0} w_{1} w_{2}} .
$$

Hence, (15) reads

$$
\begin{aligned}
\operatorname{deg}(\mathcal{F}) & =K_{\mathcal{F}} \cdot H+H . H=\int_{H}^{o r b} c_{1}\left(\mathcal{O}_{\mathbb{P}_{w}}(d-1)\right)+\int_{H}^{o r b} c_{1}\left(\mathcal{O}_{\mathbb{P}_{w}}(1)\right) \\
& =\int_{\mathbb{P}_{w}}^{o r b} c_{1}\left(\mathcal{O}_{\mathbb{P}_{w}}(d-1)\right) \wedge c_{1}\left(\mathcal{O}_{\mathbb{P}_{w}}(1)\right)+\int_{\mathbb{P}_{w}}^{o r b} c_{1}\left(\mathcal{O}_{\mathbb{P}_{w}}(1)\right) \wedge c_{1}\left(\mathcal{O}_{\mathbb{P}_{w}}(1)\right) \\
& =\frac{d-1}{w_{0} w_{1} w_{2}}+\frac{1}{w_{0} w_{1} w_{2}}=\frac{d}{w_{0} w_{1} w_{2}} .
\end{aligned}
$$

Now suppose $C$ is a quasi-smooth curve in $\mathbb{P}_{w}$, that is, is defined by a quasihomogeneous polynomial $P\left(z_{0}, z_{1}, z_{2}\right)$, of degree $d^{o}$, whose only singularity is at $0 \in \mathbb{C}^{3}$. In particular, $C$ is smooth at the orbifold points it goes through. In this case we have Kollár-Mori's adjunction formula since $C$ is a Cartier divisor in $\mathbb{P}_{w}$ (see [7, Proposition 5.73) and hence

$$
K_{C}=K_{\mathbb{P}_{w} \mid C} \otimes N_{C} .
$$

With this at hand we have, using Poincaré's duality,

$$
\operatorname{deg}(C)=\int_{C}^{o r b} c_{1}\left(\mathcal{O}_{\mathbb{P}_{w}}(1)\right)^{\operatorname{dim} C}=\int_{\mathbb{P}_{w}}^{\text {orb }} c_{1}\left(\mathcal{O}_{\mathbb{P}_{w}}\left(d^{o}\right)\right) \wedge c_{1}\left(\mathcal{O}_{\mathbb{P}_{w}}(1)\right)=\frac{d^{o}}{w_{0} w_{1} w_{2}}
$$




\section{Proof of the theorem}

Suppose $C$ is quasi-smooth, avoids the singularities of $\mathbb{P}_{w}$ and is $\mathcal{F}$-invariant.

The sum of the Camacho-Sad indices, $C S(\mathcal{F}, C)$, over $C \cap \operatorname{Sing}(\mathcal{F})$ satisfies (see [3])

$$
C S(\mathcal{F}, C)=\sum_{p \in C \cap \operatorname{Sing}(\mathcal{F})} C S(\mathcal{F}, C, p)=C . C,
$$

and, since the adjunction formula (18) holds, we have

$$
C . C=\frac{d^{\circ}}{w_{0} w_{1} w_{2}}>0
$$

so that $C \cap \operatorname{Sing}(\mathcal{F}) \neq \emptyset$. On the other hand, by (8) and (18),

$$
\begin{aligned}
& \int_{C}^{o r b} c_{1}\left(T C \otimes \mathcal{O}_{\mathbb{P}_{w}}(d-1)\right) \\
& =\frac{d^{o}\left(w_{0}+w_{1}+w_{2}-d^{o}\right)}{w_{0} w_{1} w_{2}}+\frac{(d-1) d^{o}}{w_{0} w_{1} w_{2}} \\
& =d^{o} \frac{w_{0}+w_{1}+w_{2}-d^{o}-1+d}{w_{0} w_{1} w_{2}} .
\end{aligned}
$$

Now, $\mathcal{F}_{\mid C}$ induces a nonzero holomorphic section of $T C \otimes \mathcal{O}_{\mathbb{P}_{w}}(d-1)$, and the number in (22) is the degree of this line $\mathbb{Q}$-bundle. Since $C \cap \operatorname{Sing}(\mathcal{F})$ is nonempty and finite, this degree is positive and it follows that $\operatorname{deg}(C) \leq \operatorname{deg}(\mathcal{F})+\frac{|w|-2}{w_{0} w_{1} w_{2}}$.

\section{ACKNOWLEDGEMENT}

The authors thank the referee for useful suggestions and comments.

\section{REFERENCES}

1. D. Cerveau and A. Lins Neto, Holomorphic foliations in $\mathbb{P}_{\mathbb{C}}^{2}$ having an invariant algebraic curve, Ann. Inst. Fourier 41 (1991), 883-903. MR1150571 (93b:32050)

2. M. Brunella and L. G. Mendes, Bounding the degree of solutions to Pfaff equations, Publ. Mat. 44 (2000), no. 2, 593-604. MR1800822 (2002b:32048)

3. M. Brunella, Birational Geometry of Foliations, First Latin American Congress of Mathematicians, IMPA (2000). MR1948251 (2004g:14018)

4. M. Brunella, Foliations on complex projective surfaces, Dynamical Systems, Part II, pp. 4977, Scuola Norm. Sup. Pisa, 2003. MR2071237 (2005g:32045)

5. M.M. Carnicer, The Poincaré problem in the nondicritical case, Ann. of Math. (2) 140 (1994), 289-294. MR1298714 (95k:32031)

6. E. Esteves and S. L. Kleiman, Bounding solutions of Pfaff equations. Special issue in honor of Steven L. Kleiman. Comm. Algebra 31 (2003), no. 8, 3771-3793. MR2007384 (2004m:32061)

7. J. Kollár and S. Mori, Birational Geometry of Algebraic Varieties, Cambridge Tracts in Mathematics 134, Cambridge University Press (1998). MR1658959 (2000b:14018)

8. A. Lins Neto, Some examples for the Poincaré and Painlevé problems, Ann. Scient. École Norm. Sup. $4^{e}$ série, t. 35 (2002), 231-266. MR1914932 (2003j:34009)

9. E. Mann, Cohomologie quantique orbifold des espaces projectifs à poids, J. Algebraic Geom. 17 (2008), 137-166. MR2357682 (2008k:14106)

10. H. Poincaré, Sur l'intégration algébrique des équations différentielles du premier ordre et du premier degré, Rend. Circ. Mat. Palermo 5 (1891), 161-191. 
11. J. V. Pereira, On the Poincaré problem for foliations of general type, Math. Ann. 323 (2002), no. 2, 217-226. MR1913040 (2003e:32056)

12. I. Satake, The Gauss-Bonnet theorem for V-manifolds, J. Math. Soc. Japan 9, n. 4 (1957), 464-492. MR0095520(20:2022)

13. M. G. Soares, The Poincaré problem for hypersurfaces invariant by one-dimensional foliations, Inventiones Mathematicae 128 (1997), 495-500. MR.1452431 (99a:32043)

Departamento de Matemática, Universidade Federal de Viçosa, Av. P. H. Rolfs, 36571-000 ViÇOSA, BRASIL

E-mail address: mauricio.correa@ufv.br

Departamento de Matemática, Instituto de Ciências Exatas, Universidade Federal de Minas Gerais, Av. Antonio Carlos 6627, 31270-901 Belo Horizonte, Brasil

E-mail address: msoares@mat.ufmg.br 